\title{
Strain-induced structural, magnetic and ferroelectric properties of heterostructure BST-NZFO nanocomposite thin film at room temperature
}

\author{
R K KOTNALA ${ }^{1}$, G S ARYA ${ }^{2, *}$, J YOGIRAJ ${ }^{3}$ and N S NEGI ${ }^{3}$ \\ ${ }^{1}$ National Physical Laboratory, New Delhi 110012, India \\ ${ }^{2}$ Department of Physics, Maharaja Agrasen University, Baddi 174103, India \\ ${ }^{3}$ Department of Physics, Himachal Pradesh University, Shimla 171005, India \\ *Author for correspondence (ghanshyam.phy@ gmail.com)
}

MS received 29 July 2016; accepted 24 October 2016; published online 25 July 2017

\begin{abstract}
Heterostructure $\mathrm{Ba}_{0.7} \mathrm{Sr}_{0.3} \mathrm{TiO}_{3}-\mathrm{Ni}_{0.8} \mathrm{Zn}_{0.2} \mathrm{Fe}_{2} \mathrm{O}_{4}$ composite thin films grown on $\mathrm{Pt}-\mathrm{TiO}_{2}-\mathrm{SiO}_{2}-\mathrm{Si}$ substrate were prepared by chemical solution process, where $\mathrm{Ba}_{0.7} \mathrm{Sr}_{0.3} \mathrm{TiO}_{3}$ layer grew as top/bottom while $\mathrm{Ni}_{0.8} \mathrm{Zn}_{0.2} \mathrm{Fe}_{2} \mathrm{O}_{4} l_{\text {layer }}$ grew as bottom/top. Structural characterization by X-ray diffraction and atomic force microscopy showed the similar crystal structure, different lattice parameters, large lattice strain and small grain size in heterostructures, whatever their deposition sequences. Such heterostructures present simultaneously ferromagnetic and ferroelectric responses at room temperature. In particular, an exceptionally large saturation magnetization was observed in one heterostructures film. The growth sequences of $\mathrm{Ba}_{0.7} \mathrm{Sr}_{0.3} \mathrm{TiO}_{3}$ and $\mathrm{Ni}_{0.8} \mathrm{Zn}_{0.2} \mathrm{Fe}_{2} \mathrm{O}_{4}$ layers on the substrate remarkably affect the magnetic properties of the composite thin films at room temperature.
\end{abstract}

Keywords. Nanostructured thin films; magnetic multilayer; ferroelectrics.

\section{Introduction}

Multiferroics materials, which simultaneously exhibit ferroelectricity and ferromagnetism, have recently stimulated increasing interest for their significant potential applications in many advanced multifunctional devices [1-5]. Among them, a tremendous flurry of research activities have been reported on multiferroics thin films in the past few years, which have been grown on different substrates by different experimental techniques [5-8]. Motivated by a pioneering work of many groups, multiferroics nanocomposites of ferroelectric and ferromagnetic oxides have become new routes to many multiferroics composites [7-10]. In comparison with bulk multiferroic composites, the nanostructured thin films provide more degrees of freedom such as lattice strain or interlayer interaction, to modify the multiferroics behaviour. The lattice strain at the interface of composite-layered thin films plays a crucial role in the enhancement of magnetoelectric coupling between the composites phases. Recently, strain-induced magnetoelectric properties were discovered in many materials, which allow electric field control of magnetic properties [11-13]. In pursuit of achieving the highest magnetoelectric coupling in composite multiferroic thin films, different composites nanostructures such as $0-3$ particulate composite (bulk), 2-2 laminates composite and 1-3 fibrerod composite films have been investigated to a large extent [5-8]. Among them, the 2-2 type heterostructure thin films, consisting of alternating layer of ferroelectric perovskite and magnetic oxides, have many advantages over conventional composite nanostructures, including their easy synthesis, better mutual control of different layers, and most important, small leakage current density by significantly isolating the resistive magnetic layer with insulating ferroelectric layers. Therefore, the 2-2 type heterostructure composites are promising and potential candidates for multiferroics applications.

In this work, we report a heterostructure composite thin film of $\mathrm{Ba}_{0.7} \mathrm{Sr}_{0.3} \mathrm{TiO}_{3}$ (BST) and $\mathrm{Ni}_{0.8} \mathrm{Zn}_{0.2} \mathrm{Fe}_{2} \mathrm{O}_{4}$ (NZFO) (2-2 type structure) with different growth sequences on a $(1500 \AA) \mathrm{Pt}(111)-(400 \AA) \mathrm{TiO}_{2}-(0.5 \mu \mathrm{m}) \mathrm{SiO}_{2}-\mathrm{Si}$ substrate. BST is one of the most studied ferroelectric materials in the perovskite family due to its high figure of merit, environment friendly nature and large ferroelectric properties at room temperature [14]. NZFO has a few advantages such as large resistivity, high saturation magnetization and much smaller coercive field [15], which are helpful in improving the magnetic properties of composite thin films, and hence offers high magnetoelectric coupling in these materials.

To date, no research has been made on BST and NZFO composite $2-2$ heterostructure prepared by metal-organic decomposition (MOD) method (NZFO) and sol-gel technique (BST). In addition, the strain-induced magnetic properties in composite thin films are also an interesting area of research activity. Therefore, the effect of strain-mediated 
improvement on the structural, magnetic and ferroelectric properties due to different growth sequences of BST and NZFO phases on Si substrate has been discussed in the present work.

\section{Experimental}

\subsection{Materials and methods}

BST- $\mathrm{Ni}_{0.7} \mathrm{Zn}_{0.3} \mathrm{Fe}_{2} \mathrm{O}_{3}$ composite thin films were grown on (1500 ̊) Pt (111)-(400 ̊) $\mathrm{TiO}_{2}-(0.5 \mu \mathrm{m}) \mathrm{SiO}_{2}-\mathrm{Si}$ substrate using MOD/chemical solution deposition-derived spin coating technique, from two stoichiometric BST (sol-gel method) and NZFO (MOD technique) single phases. The deposition temperature was first kept at $400^{\circ} \mathrm{C}$ for the growth of different BST-NZFO layers, and final sintering was performed at $750^{\circ} \mathrm{C}$ for $2 \mathrm{~h}$. The total number of layers was six, three of ferroelectric phases and other three of ferrite phases grown simultaneously on Si substrate as per their required sequence. This was done to obtain the minimum thickness of each sample. After the deposition, the samples were cooled to room temperature at a cooling rate of $10^{\circ} \mathrm{C} \mathrm{min}^{-1}$. A flowchart showing detailed steps for the synthesis of NZFO-Si thin films is shown in figure 1 .

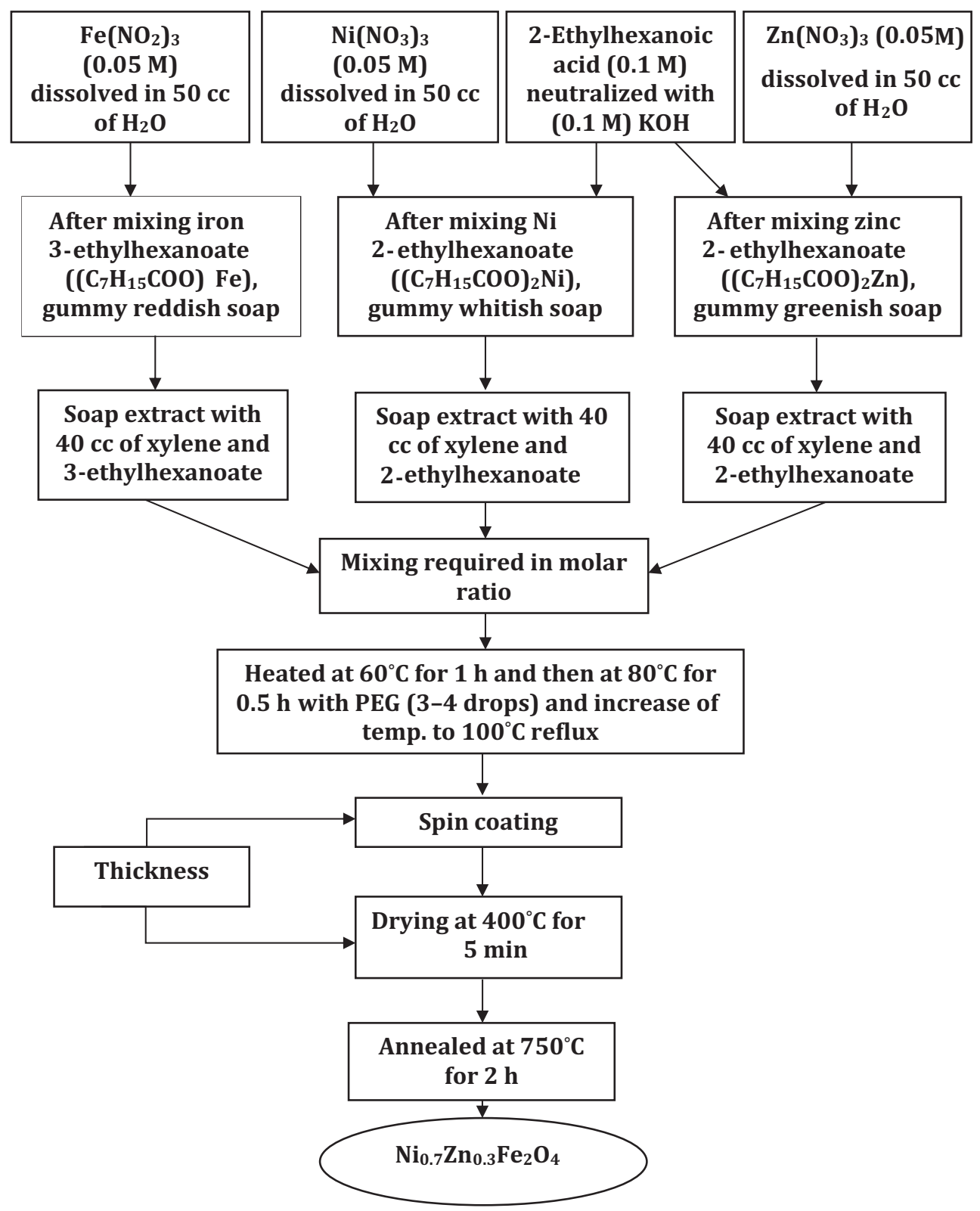

Figure 1. Flowchart representation of synthesis of NZFO-Si by MOD technique. 


\subsection{Characterization}

The phase analysis of the films was performed by X-ray diffraction (XRD) with $\mathrm{Cu} \mathrm{K} \alpha$. An atomic force microscope (AFM) was utilized for the observation of the composite thin film microstructures.

For the electrical measurements, Al electrode dots were deposited on the composite film surface by DC sputtering techniques. The magnetic measurements of these composite films were performed using a vibratory sample magnetometer (VSM). Ferroelectric properties of films were measured using a Precision multiferroic analyser. All measurements were performed at room temperature.

\section{Results and discussion}

\subsection{XRD analysis}

Typical XRD patterns of the different composite thin films are shown in figure 2. The XRD patterns of BST-Si and NZFO$\mathrm{Si}$ thin films in the present work are in agreement with earlier reported results on these materials $[14,15]$. The BST-NZFOSi film and its reversal heterostructure NZFO-BST-Si have similar XRD patterns irrespective of their growth sequence on Si substrate. The XRD patterns clearly illustrate that each layer in composite thin films has its crystalline orientation and diffraction peaks corresponding to only BST and NZFO phases are observed.

In the BST-NZFO-Si composite film, the bottom NZFO layer has the smallest lattice parameters calculated from XRD measurement, while the largest lattice parameter for NZFO is in the NZFO-BST-Si film, in comparison with NZFO-Si film (table 1). Therefore, larger lattice distortion occurs in the BST-NZFO-Si film because the bottom NZFO layer is constrained from two sides. However, the compressive strains in the NZFO layer are almost released in the NZFO-BST$\mathrm{Si}$ film, and in this case, the bottom BST layer acts as a buffer layer that effectively reduces constrains from Si substrate. The presence of large lattice strain and smaller grains in composite thin films significantly contribute to broadening of characteristics peaks. The diffraction peak broadening generally arises from the presence of small-size grains and large lattice strain $[16,17]$. The presence of broader characteristics peaks, e.g. (511), in the ferrite phase of BST-NZFO-Si composites therefore supports the large lattice strain and small crystallite size.

The value of crystallite size for NZFO phase in different composites is calculated by applying Scherer's formula [16]. The calculated values of crystallite size for NZFO phase
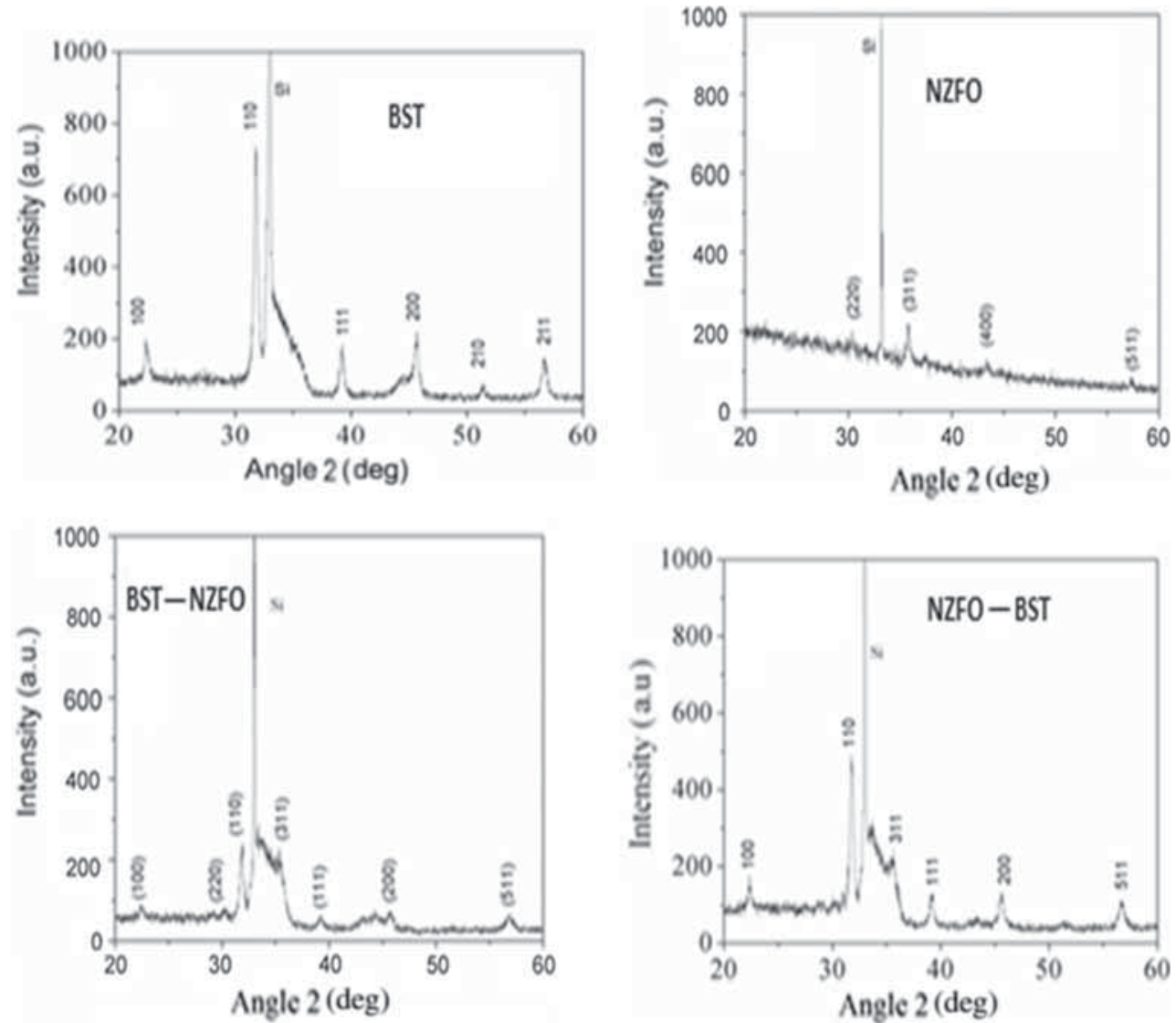

Figure 2. XRD patterns of different nanocomposite thin films. 
Table 1. Lattice constants, grain size $(d)$, saturation magnetization $\left(M_{\mathrm{S}}\right)$ and saturation polarization $\left(P_{\mathrm{S}}\right)$ of various samples.

\begin{tabular}{|c|c|c|c|c|c|c|}
\hline \multirow[b]{2}{*}{ Sample } & \multicolumn{2}{|c|}{ Lattice constants } & \multirow[b]{2}{*}{$c / a$ ratio } & \multirow[b]{2}{*}{$d(\mathrm{~nm})$} & \multirow[b]{2}{*}{$M_{\mathrm{S}}\left(\mathrm{emu} \mathrm{g}^{-1}\right)$} & \multirow[b]{2}{*}{$P_{\mathrm{S}}\left(\mu \mathrm{Ccm}^{-2}\right)$ at $15 \mathrm{~V}$} \\
\hline & $a(\AA)$ & $c(\AA)$ & & & & \\
\hline BST-Si & 3.98 & 3.99 & 1.003 & 40 & - & 18 \\
\hline NZFO-Si & $\begin{array}{l}8.33 \\
\text { Ferrite }\end{array}$ & $\overline{\text { Ferroelectric }}$ & $\begin{array}{l}1 \\
\text { Ferroelectric }\end{array}$ & 12 & 142 & - \\
\hline NZFO-BST-Si & 8.39 & $a=3.96, \quad c=3.99$ & 1.007 & 16 & 96 & 16 \\
\hline BST-NZFO-Si & 8.32 & $a=4.06, \quad c=4.11$ & 1.012 & 7 & 214 & 17 \\
\hline
\end{tabular}

are 16, 12 and $7 \mathrm{~nm}$ in NZFO-BST-Si, NZFO-Si and BSTNZFO-Si samples, respectively.

The crystallite size and lattice strain $(\varepsilon)$ induced on ferrite phase by the BST layer and Si substrate for different thin films can be calculated using the Williamson-Hall equation $[17,18]$ :

$$
\beta \cos \theta=4 \varepsilon \sin \theta+k \lambda / d,
$$

where $d$ is crystallite size, $\lambda$ is wavelength of $\mathrm{X}$-rays (1.5405 $\AA$ ), $\beta$ is half-width of XRD peaks in radian, $k$ is shape factor (0.9) and $\theta$ the angle of reflection. In deriving equation (1) it is assumed that strain is uniform in all crystallographic directions, which is known as the uniform deformation model. In this model the crystal is considered as isotropic in nature and properties of material are independent of the direction along which it is measured. The values of $\beta \cos \theta$ on $y$-axis were plotted as a function of $4 \sin \theta$ on $x$-axis, and the crystalline size $d$ was estimated from the $y$ intercept, and the microstrain $\varepsilon$ from the slope of the graph. Figure 3 shows the $\beta \cos \theta$ vs. $4 \sin \theta$ graph for the three composites. The grain size calculated from the graphs almost match well with the one calculated using the Scherer formula for the most intense peak of NZFO phase. The strain values calculated from the linear fit of the graphs are 0.0042, 0.0089 and 0.012 for NZFO-BST-Si, NZFO-Si and BST-NZFOSi samples, respectively. The variation of crystallite size and lattice strain for different samples is shown in figure 4. It is noted that a very small crystallite size and high value of lattice strain are observed for NZFO phase in BST-NZFO-Si thin film, which can be attributed to large compressive strain from BST layer and Si substrate that subsequently plays an important role in the enhancement of its magnetic properties.

As for the BST, the bottom BST layer in the NZFO-BSTSi thin film suffers compressive strain from Si substrate, and a small lattice distortion is observed. In contrast, the top BST layer in BST-NZFO-Si film suffers a tangible strain from NZFO layer with large lattice parameters, and in this case, large lattice distortion is observed (table 1). Overall, the decrease in lattice parameters for ferrite phase in BSTNZFO-Si is attributed to large internal lattice strain and large surface energy effect of small nanoparticles on its surface. In contrast, these effects produce structural distortion in ferroelectric phase of this sample, which contributes to its ferroelectric properties.

\subsection{Surface morphology}

Figure 5 shows AFM images of these composite thin films. It can be seen from different images that the microstructure of films has different nanoparticles growth sequences and shows granular structure, and average grains size varies from 7 to $40 \mathrm{~nm}$ (table 1). The average roughness is in the range of $1-12 \mathrm{~nm}$, and generally increases with increasing grain size. The surface morphology of BST-NZFO-Si composite film presents the least developed grain structure coupled with porous network, which promotes the formation of nonbridging-type oxygen defect at the interface of composite layers.

\subsection{Magnetic properties}

The variation of magnetization as a function of applied magnetic field measured at room temperature is shown in figure $6 \mathrm{a}$. As shown in figure $6 \mathrm{~b}$, the saturation magnetizations for the NZFO-Si and BST-NZFO-Si films are 142 and $96 \mathrm{emu} \mathrm{cm}{ }^{-3}$, respectively, while the composite-phase BSTNZFO-Si thin film has higher saturation magnetization (i.e., $244 \mathrm{emu} \mathrm{cm}^{-3}$ ) than that of NZFO-Si film. Generally the magnetic properties in NZFO-BST-Si and BST-NZFO-Si composite films emerge due to connection of ferroelectric grain with neighbouring ferrite grain. The observation of large magnetization in BST-NZFO-Si composite thin film is an extraordinary finding and is mainly attributed to the presence of smaller particles, large strain and formation of defect states at the interface of composite phase [10-16]. As the particles size in ferrite phase of BST-NZFO-Si is smaller, the surface effect becomes dominant, i.e., as the volume to surface ratio of particles decreases, the uncompensated surface magnetic spin induced by the confinement of small nanoparticles significantly contributes to improved magnetic properties [17-20]. The improved magnetic properties of this sample can also be related to the presence of large lattice strain. The maximum 

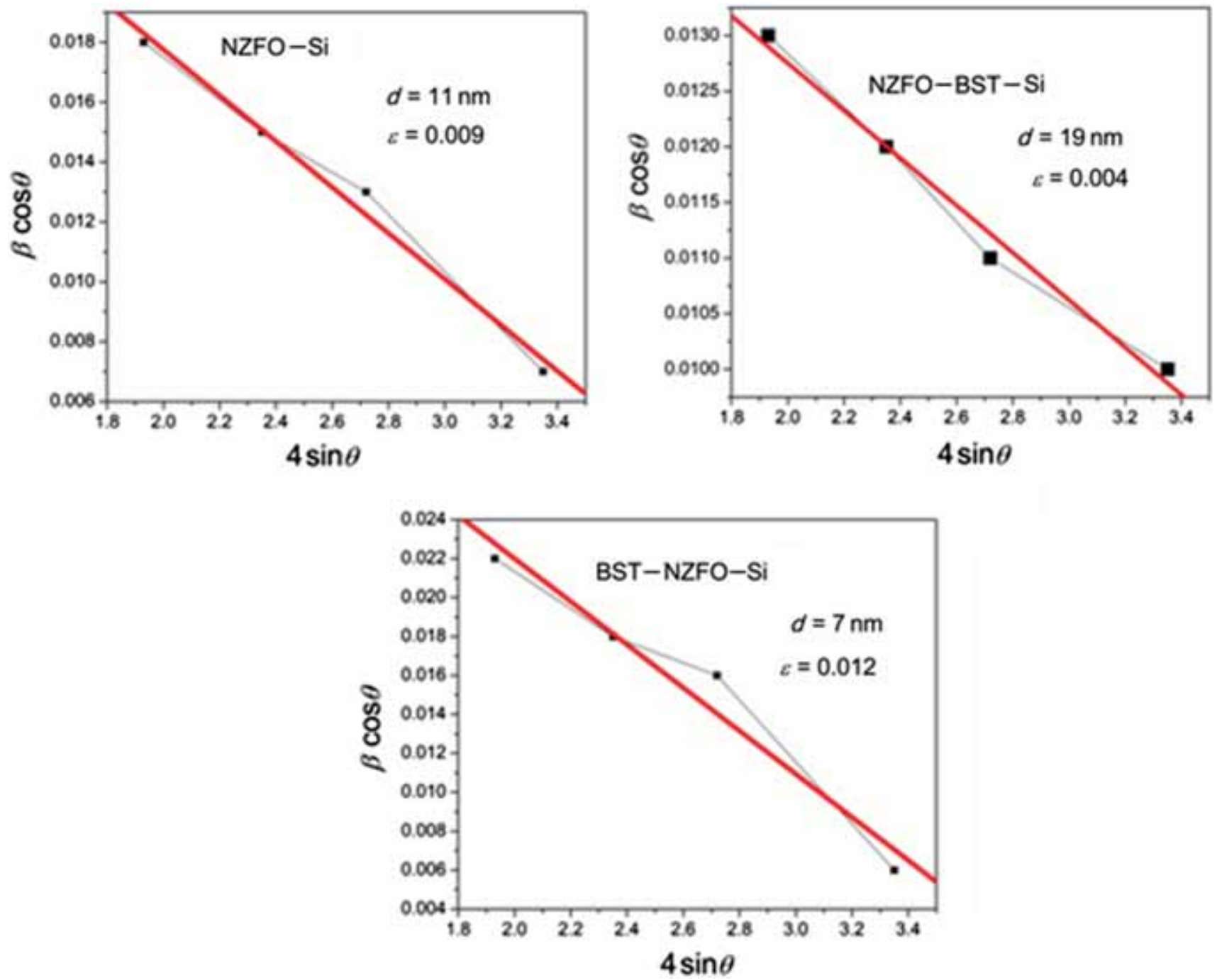

Figure 3. $\beta \cos \theta$ vs. $4 \sin \theta$ plot for different composites series.

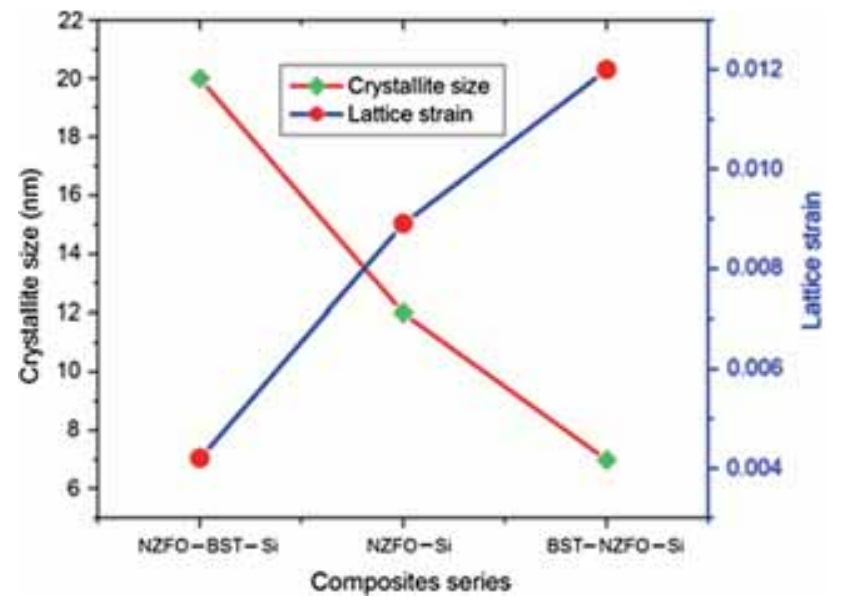

Figure 4. Variation of crystallites size and lattice strain for different composites thin films. strain associated with small nanoparticles produces a disordered structure by displacements of ions, which is another cause for large magnetization. As most of the atoms are present on the surface, the surface effect as well as the change in position of cations and anions will promote an enhancement of magnetic properties. The effect of strain on the improvement of magnetic properties in the present work also finds support from strain-induced magnetic improvement investigated earlier in many materials [19-22]. The defects states as indicated by AFM images such as oxygen vacancies can also increase magnetization as the presence of defect states generally promotes the super-exchange interaction between adjacent $\mathrm{Fe}$ ions in different sublattices, leading to large magnetization; however, measurements like XPS may be needed to establish this fact.

In contrast, the coercive field for these films decreases in the order 70, 50 and 38 Oe for the NZFO-Si, NZFO-BST-Si 

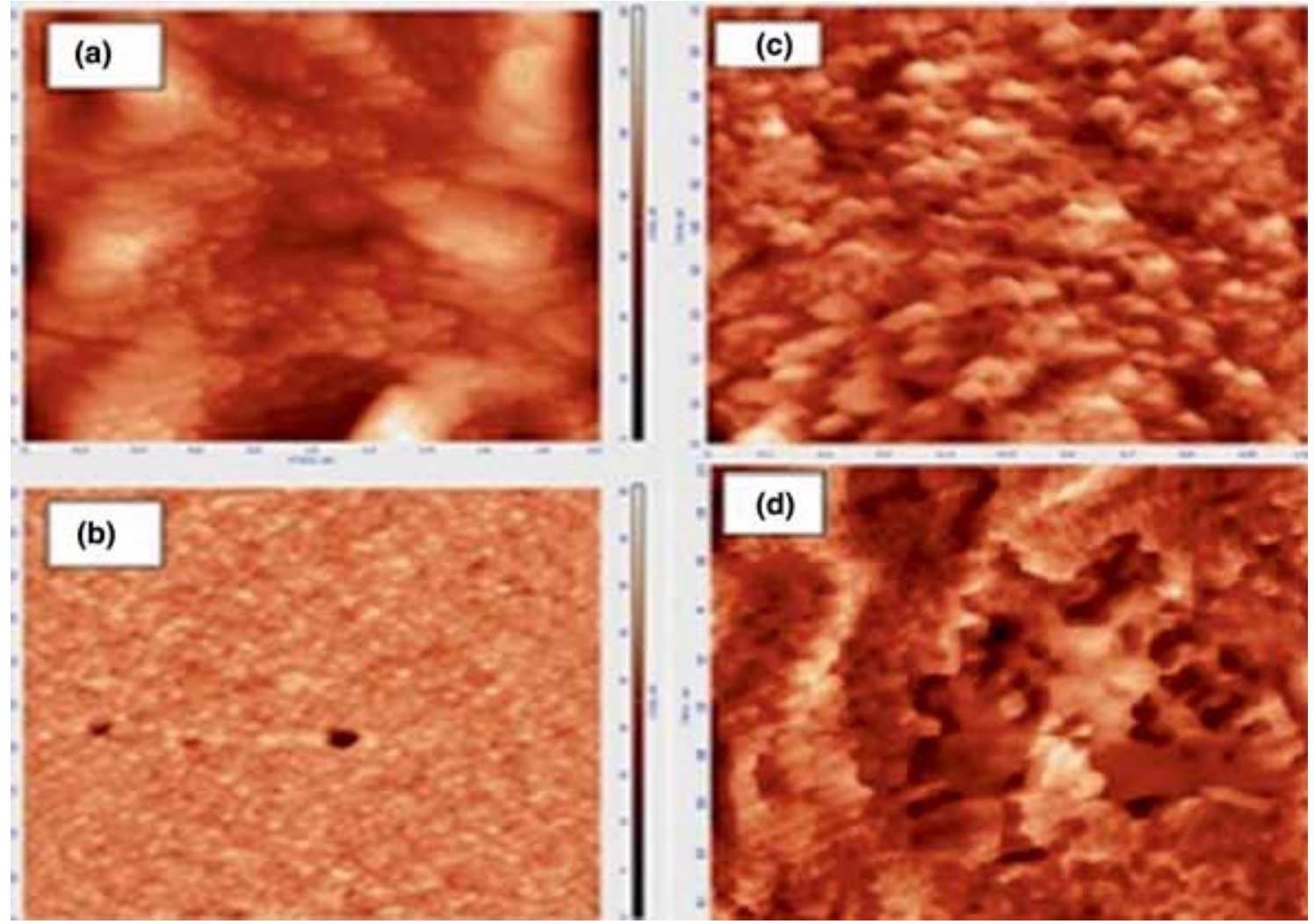

Figure 5. AFM images of (a) BST-Si, (b) NZFO-Si, (c) NZFO-BST-Si and (d) BST-NZFO-Si thin film.

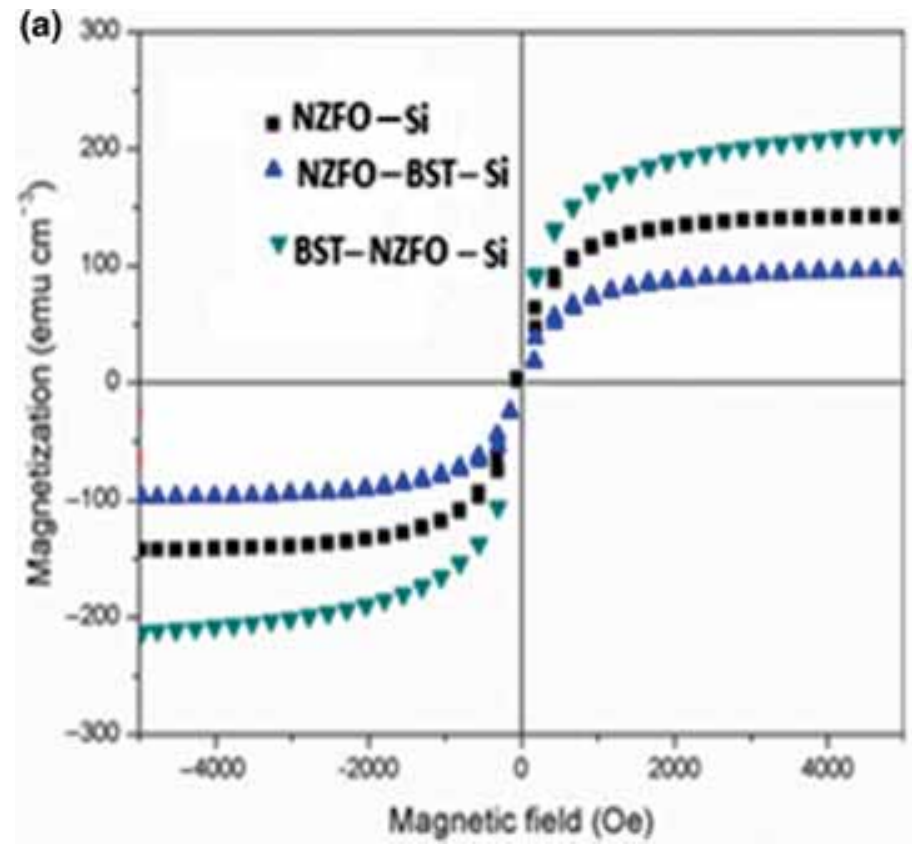

(b)

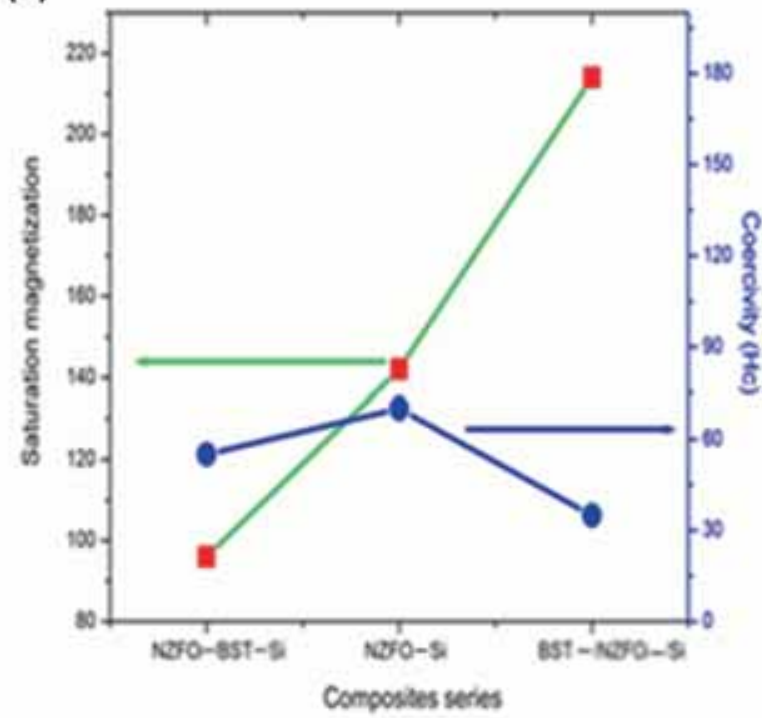

Figure 6. (a) $M-H$ curves of different thin films measured; (b) variation of saturation magnetization and coercivity.

and BST-NZFO-Si, respectively (figure 6b). The decrease in coercivity is mostly related with the presence of smallsize particles in BST-NZFO-Si sample. The small particles mostly prefer single-magnetic-domain structure, which leads to the appearance of almost super-paramagnetic-type $M-H$ curves. The origin of small coercivity in the BST-NZFO-Si 

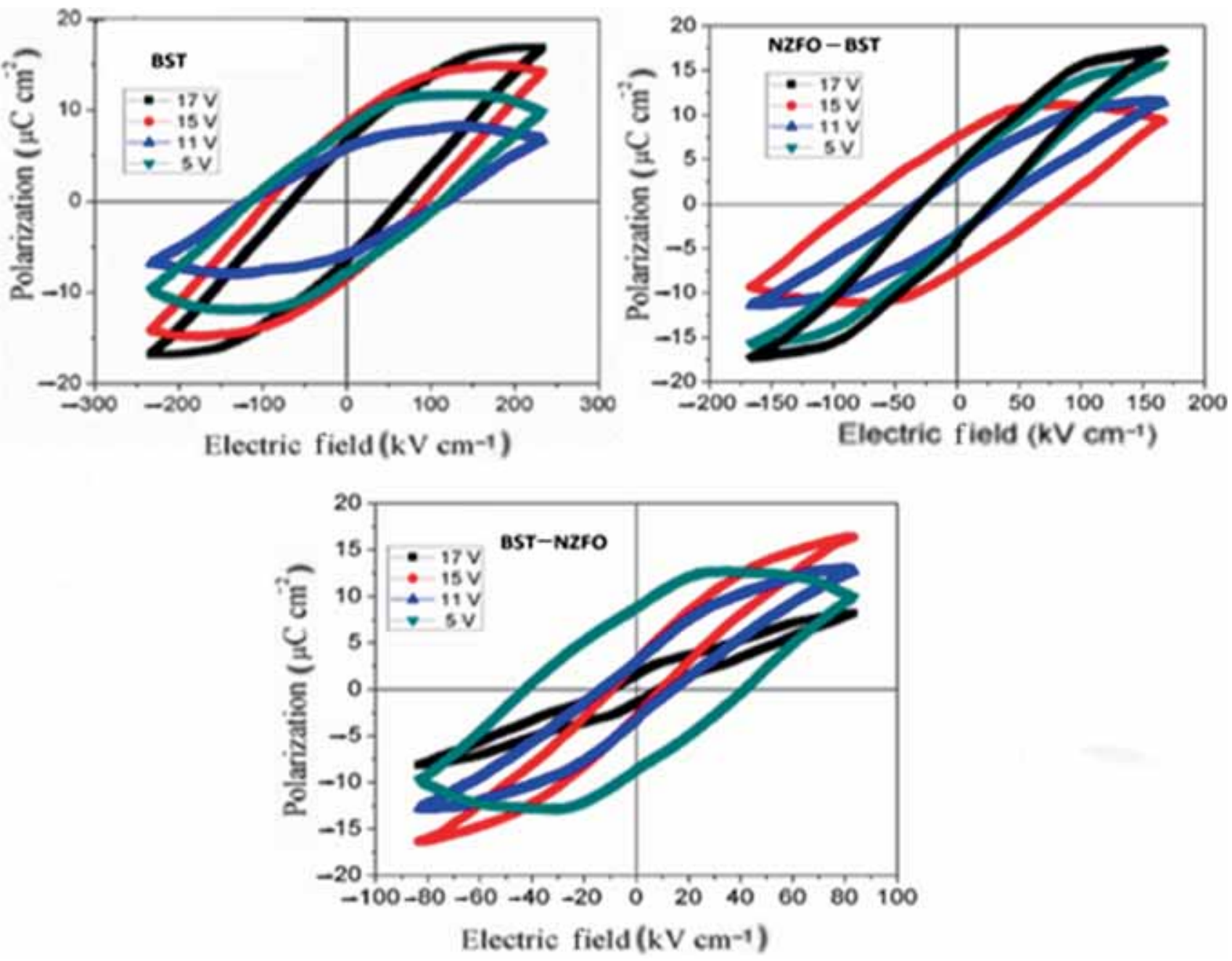

Figure 7. $P-E$ hysteresis loop of BST-Si, NZFO-BST-Si and BST-NZFO-Si at different switching voltages.

composite film is attributed to decrease in the magnetocrystalline anisotropy as a consequence of size effect $[4,23]$.

\section{$3.4 \quad$ Ferroelectric properties}

The ferroelectric hysteresis loops for these thin films measured at different switching voltages are shown in figure 7. The NZFO-BST-Si and BST-NZFO-Si composite thin films exhibit significant ferroelectric hysteresis loops like the BST-Si single-phase film. The saturation polarization of these composite samples is almost equal to that of BSTSi single phase film $\left(18 \mu \mathrm{Ccm}^{-2}\right)$ at $15 \mathrm{~V}$, which indicates that BST layer in these composite thin films has a high quality.

As shown in figure 7, the shape of loops is a function of applied voltage. In BST-Si, as the voltage increases, the loops become slim with net enhancement of saturation polarization. On the other hand, the shape of loops in NZFO-BST-Si becomes flat with increasing voltage, which means that the role of defects in generating polarization seems visible as voltage increases. In BST-NZFO-Si, the shape of $P-E$ loops remains slim up to $15 \mathrm{~V}$, and thereafter, an anomalous hysteresis loop (leaky loop) at $17 \mathrm{~V}$ is observed. In perovskite $\mathrm{ABO}_{3}$-type materials, oxygen loss generally occurs during the sintering process and can be represented by the KrogerVink notation as

$$
\mathrm{Oo} \rightarrow 1 / 2 \mathrm{O}_{2}+\mathrm{Vo}+2 \mathrm{e}
$$

Electrons released due to oxygen vacancy Vo may be captured by $\mathrm{Fe}^{3+}$ of BST-NZFO-Si, leading to its reduction to $\mathrm{Fe}^{2+}$. The presence of $\mathrm{Fe}^{2+}$ and $\mathrm{Fe}^{3+}$ ions would lead to hopping of electrons, which increases conductivity and consequently appearance of a leaky $P-E$ loop.

\section{Conclusions}

In summary, heterostructures BST-NZFO composite thin films have been grown on $\mathrm{Pt}-\mathrm{TiO}_{2}-\mathrm{SiO}_{2}-\mathrm{Si}$ substrate by 
chemical deposition method. Structural characterization by XRD and atomic force microscopy showed similar crystal structure, different lattice parameters mediated through strain and small grain size in heterostructures irrespective of their deposition sequences. Such heterostructures present simultaneously ferromagnetic and ferroelectric responses at room temperature. The BST-NZFO-Si composite has a large saturation magnetization and good ferroelectric properties, which make it a suitable candidate for many technologically advanced electronic devices.

\section{Acknowledgement}

J Yogiraj acknowledges no financial support in carrying out this work.

\section{References}

[1] Ramesh R and Spaldin N A 2007 Nat. Mater. 621

[2] Li Y, Shu L, Huang W, Jiang X and Wang H 2014 Appl. Phys. Lett. 105162906

[3] Wang J, Neaton J B, Zheng H, Nagrajan V, Ogale S B, Liu B et al 2003 Science 2991719

[4] Arya G S and Negi N S 2013 J. Phys. D: Appl. Phys. 46095004

[5] Barbier A, Aghavnian T, Badjeck V, Mocuta C, Stanescu D, Magnan $\mathrm{H}$ et al 2015 Phys. Rev. B 91035417
[6] Schröder J, Labusch M, Keip M A, Kiefer B, Brands D and Lupascu D C 2015 GAMM Mitt. 388

[7] Lorenz M, Wagner G, Lorenza V, Schwinkendorf P, Modarresi H, Van Bael M J et al 2015 Appl. Phys. Lett. 106012905

[8] Nan C W, Bichurin M I, Dong Shuxiang, Viehland D and Srinivasan G 2008 J. Appl. Phys. 103031101

[9] Islam A and Priya S 2006 Appl. Phys. Lett. 89152911

[10] Zippel J, Lorenz M, Setzer A, Rothermel M, Spemann D, Esquinazi P et al 2013 J. Phys. D: Appl. Phys. 46275002

[11] Yang W G, Morley N A, Sharp J, Tian Y and Rainforth W M 2016 Appl. Phys. Lett. 108012901

[12] Gorige E, Kati R, Yoon D H and Kuma P S A 2016 J. Phys. D: Appl. Phys. 49405001

[13] Chen A T and Zhaoa Y G 2016 APL Mater. 4032303

[14] Gust M C, Momoda L A, Evans N D and Mecartney M L 2001 J. Am. Ceram. Soc. 841087

[15] Liu Y, Li Y, Zhang H, Chen D and Mu C 2011 J. Appl. Phys. 109 07A511

[16] Shenoya S D, Joy P A and Anantharamana M R 2004 J. Magn. Magn. Mater. 269217

[17] Bindu P and Thomas S 2014 J. Theor. Appl. Phys. 8123

[18] Zak A K, Majid W H A, Abrishami M E and Yousefi R 2011 Solid State Sci. 13251

[19] Arya G S, Sharma R K and Negi N S 2013 Mater. Lett. 93341

[20] Smolenski T, Kazimierczuk T, Kobak J, Goryca M, Golnik A, Kossacki P et al 2016 Nat. Commun. 710484

[21] Lee J, Lauhoff G, Tselepi M, Hope S, Rosenbusch P, Bland J A C et al 1997 Phys. Rev. B 5522

[22] Zhoua W, Liub L and Wua P 2014 Phys. Lett. A 378909

[23] Arya G S, Kotnala R K and Negi N S 2013 J. Appl. Phys. 113 044107 